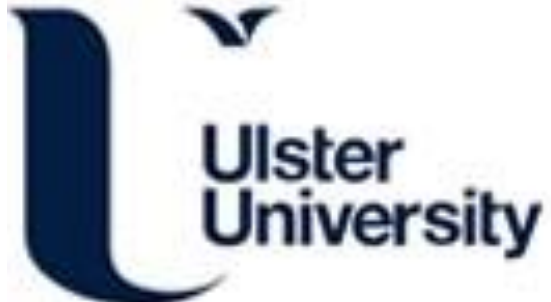

\section{A Smartphone Based Hand-Held Indoor Positioning System}

Zheng, L., Wu, Z., Zhou, W., Weng, S., \& Zheng, H. (2016). A Smartphone Based Hand-Held Indoor Positioning System. In J. Hung, N. Yen, \& K-C. Li (Eds.), Lecture Notes in Electrical Engineering: Frontier Computing (Vol. vol 375, pp. 639). Springer. https://doi.org/10.1007/978-981-10-0539-8_62

Link to publication record in Ulster University Research Portal

\section{Published in:}

Lecture Notes in Electrical Engineering

Publication Status:

Published (in print/issue): 01/01/2016

DOI:

10.1007/978-981-10-0539-8_62

\section{Document Version}

Publisher's PDF, also known as Version of record

\section{General rights}

Copyright for the publications made accessible via Ulster University's Research Portal is retained by the author(s) and / or other copyright owners and it is a condition of accessing these publications that users recognise and abide by the legal requirements associated with these rights.

\section{Take down policy}

The Research Portal is Ulster University's institutional repository that provides access to Ulster's research outputs. Every effort has been made to ensure that content in the Research Portal does not infringe any person's rights, or applicable UK laws. If you discover content in the Research Portal that you believe breaches copyright or violates any law, please contact pure-support@ulster.ac.uk. 


\section{A Smartphone Based Hand-Held Indoor Positioning System}

Chapter in Lecture Notes in Electrical Engineering · January 2016 DOI: 10.1007/978-981-10-0539-8_62

5 authors, including

Lingxiang Zheng

Xiamen University

37 PUBLICATIONS 71 CITATIONS

SEE PROFILE
Zhou Wencheng

Xiamen University

3 PUBLICATIONS 24 CITATIONS

SEE PROFILE

9. Huiru Zheng

Ulster University

256 PUBLICATIONS $\mathbf{1 , 7 6 9}$ CITATIONS

SEE PROFILE

Some of the authors of this publication are also working on these related projects: 


\title{
A Smartphone Based Hand-Held Indoor Positioning System
}

\author{
Lingxiang Zheng, Zongheng Wu, Wencheng Zhou, Shaolin Weng \\ and Huiru Zheng
}

\begin{abstract}
In this paper, we present a smartphone-based hand-held indoor positioning system. The system collects data using the accelerometer, gyroscope and gravity virtual sensor sensors embedded in the smartphone. The accelerometer and gravity data are used to detect zero vertical speed and calculate the vertical displacement of each walking step, and then the Pythagorean Theorem is applied to calculate the step length of every step. Gyroscope data is used to estimate the direction angle. The step length and the direction angle of each step is combined to determine the coordinates of each step. A Kalman filter is used to reduce the vertical speed offset caused by accelerometer drift errors. The testing results show good performance of the proposed system.
\end{abstract}

Keywords Smartphone - Indoor positioning - Kalman filter

\section{Introduction}

In many applications, indoor positioning is required, in particular where the Global Positioning System (GPS) is not available, for example, tracking a firefighter in fire scenes, looking for a car in an underground parking, tracking health-care workers and instruments in a hospital, and so on.

Currently, most indoor positioning methods use infrastructure-based approaches and rely on the signals of external devices. It limits their usability, especially when there are not any external sources of signals or these signals are hard to

\footnotetext{
L. Zheng $(\varangle) \cdot$ Z. Wu $\cdot$ W. Zhou $\cdot$ S. Weng $\cdot$ H. Zheng

School of Information Science and Engineering, Xiamen University, Xiamen, China

e-mail: lxzheng@xmu.edu.cn
}

H. Zheng

e-mail: h.zheng@ulster.ac.uk

L. Zheng $\cdot$ Z. Wu $\cdot$ W. Zhou $\cdot$ S. Weng $\cdot$ H. Zheng

School of Computing and Mathematics, Ulster University, Jordanstown Campus,

Shore Road, Newtownabbey, Co. Antrim, UK 
setup. Moreover, the wireless signal source is vulnerable to interruptions or interferences, which makes it harder to establish an accurate and stabilized indoor positioning system.

Infrastructure-free-based approaches eliminate the needs of external signals. Most of these approaches are based on accelerometers and gyroscopes. Depending on where the sensors are placed, previous studies generally could be classified into three types: foot mounted [1-8], waist mounted [9-12] and hand-held [13-15]. In our previous research $[16,17]$, we proposed a 3D indoor positioning system using low cost MEMS sensors mounted on foot, algorithms were developed to calculate the position using a Kalman filter. In [18], the author put a smart phone in pocket and estimated the vertical displacement by double integral vertical velocity, then calculate the step length through the Pythagorean Theorem, and obtained the attitude information through map matching. In [15], the author presented an activity sequence-based indoor pedestrian localization approach using smartphones held in hand in front of body. Nonetheless, these methods still use specialized sensors, require a floor plan, and have to go through a training phase or suffer from low accuracy.

To overcome the above limitations, we developed an indoor positioning system using a smartphone held on hand static to body and the system doesn't require training nor a floor plan. This method uses a smartphone application to collect the data of phone's built-in accelerometer, gyroscope and virtual gravity sensor. A 5-dimensional Kalman filter is designed in accordance with the motion equation to calculate the vertical velocity. The vertical displacement is the integral of vertical velocity, step lengths are then obtained using the Pythagorean Theorem given the length of legs. The heading of each step can be estimated by the angular velocity measured by gyroscope.

The sensors can accurately collect data in harsh environment. However, the drift and bias errors of the sensors cause serious problems. Such errors can accumulate over time, so the measured vertical acceleration over time accumulated an increasingly large error. We use a zero velocity update method (ZUPT) method to overcome the sensors errors and improve the accuracy significantly.

The rest of this paper is organized as follows: Sect. 2 introduces details of the design, framework and the processing method. Section 3 presents the experiments and results. Section 4 concludes the paper.

\section{Methods}

The overall design and the execution framework of the system is shown in Fig. 1. The system consists of five modules: data acquisition, ZUPT, step length calculation, Kalman filter, and trajectory calculation. 


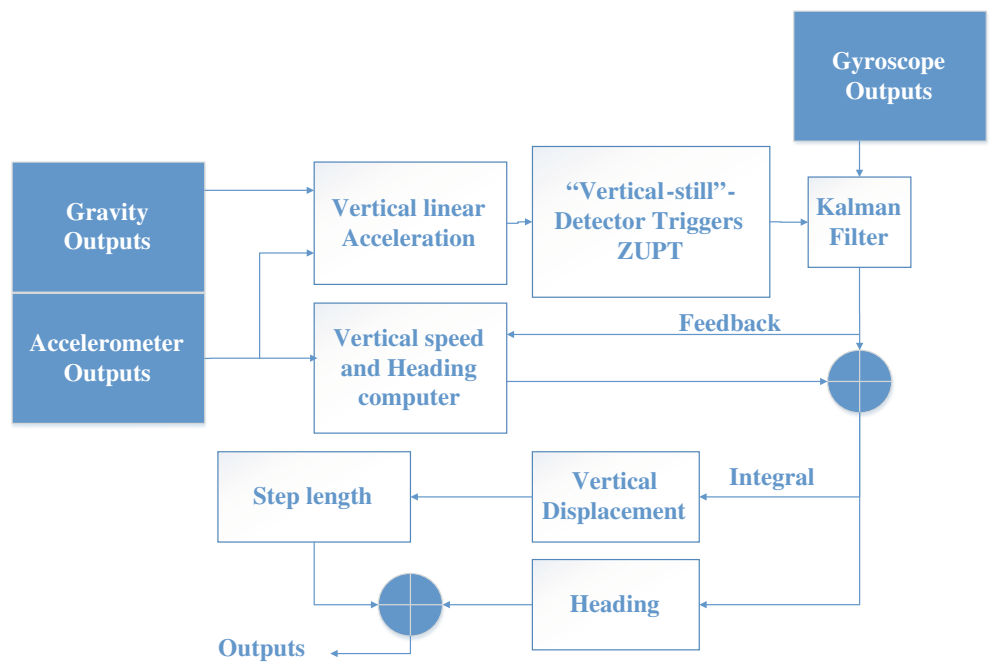

Fig. 1 System architecture

\subsection{Data Acquisition}

We designed an android APP to acquire data from accelerometers, gyroscopes and gravity sensor (virtual sensor filtered by acceleration) from the smart phone hold by the pedestrian. The sampling interval is $40 \mathrm{~ms}$. We found that sampling interval faster than $40 \mathrm{~ms}$ doesn't improve experimental accuracy, only to increase the phone's power consumption. The sampling interval slower than $40 \mathrm{~ms}$ will reduce the experimental accuracy. The outputs of the accelerometers and gravity sensor are sent to ZUPT module to detect the zero vertical velocity moments. In addition, a Kalman filter based framework is used to fusion the outputs of the sensors and to estimate the non-linear error of the vertical velocity and heading that increases over time, so the acceleration information is also sent to Kalman filter together with the outputs of the gyroscopes.

\section{$2.2 Z U P T$}

ZUPT (zero velocity update) has been proved to be an effective method to control and eliminate data drift errors. ZUPT is triggered when the vertical velocity of trunk is zero. During one step (from one heel-touching-ground event to the next heel-touching-ground event), there are two events which vertical velocity of trunk will be zero, the first one is a heel-touching-ground event, which happens when the 
heel just hits the ground and the trunk is in its lowest position during the entire step [18]. The second one is the stance, which occurs when the foot is flat on the ground and the trunk reaches its highest point. Meanwhile, while these two event happen, the linear acceleration of trunk meets its minimum and maximum points respectively. So we can extract these two zero vertical velocity moments of every step.

Gravity sensor is a virtual sensor obtained by filtering the acceleration sensor, its result is a three-dimensional vector of gravity acceleration. Taken straight up to a positive direction, according the vector dot product principle, the vertical linear acceleration $a_{z}$ is computed as (1). To extract each of the peaks and valleys will get two zero vertical velocity moments in every steps.

$$
a_{z}=-\frac{(\vec{a}-\vec{g}) \cdot \vec{g}}{|\vec{g}|}
$$

where $\vec{a}$ represents the result vector of accelerometer, $\vec{g}$ is the acceleration of gravity. The detection of zero vertical velocity is shown in Fig. 2.
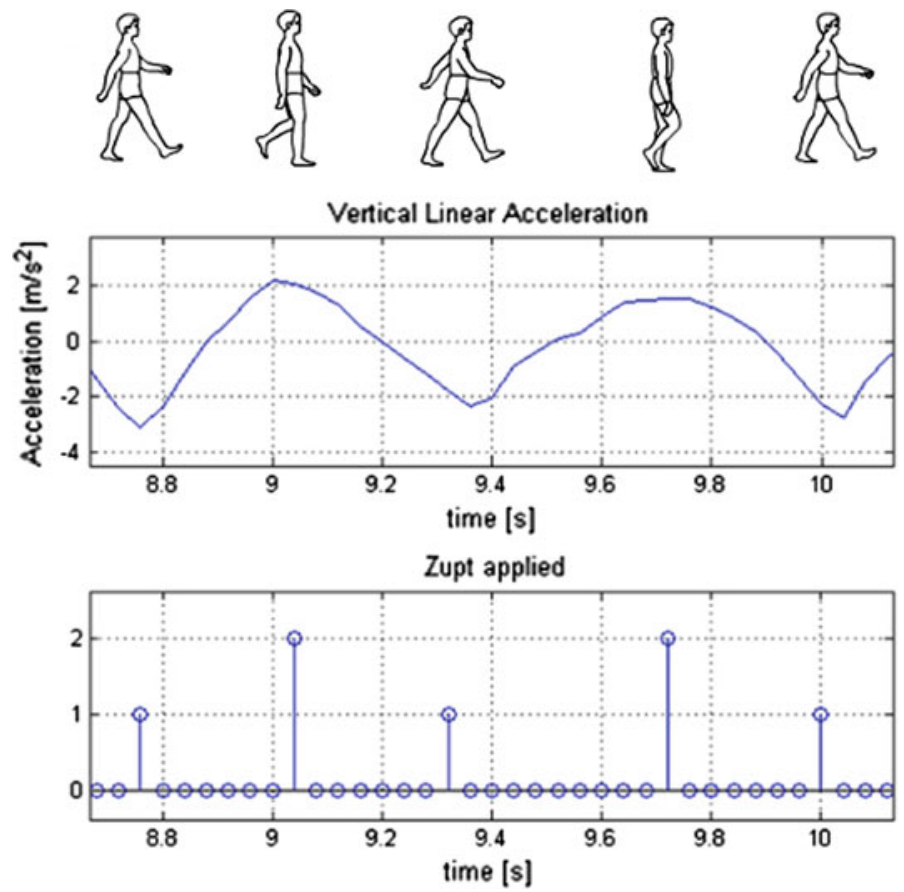

Fig. 2 Detection of zero vertical velocity 


\subsection{Kalman Filter Design}

Navigation parameters used in the study are vertical velocity, vertical displacement, and attitude information. So we built a transition model $X(k)$ which consist of vertical velocity $\left(V_{z}(k)\right)$, vertical displacement $(Z(k))$, and attitude information of the pedestrian $(\operatorname{Roll}(k), \operatorname{Pitch}(k), \operatorname{Yaw}(k))$ respectively:

$$
X(k)=\left[Z(k), V_{z}(k), \operatorname{Roll}(k), \operatorname{Pitch}(k), \operatorname{Yaw}(k)\right]
$$

We listed the equations of motion to solve the vertical velocity and vertical displacement of the transition model:

$$
\left[\begin{array}{c}
Z(k+1) \\
V_{z}(k+1)
\end{array}\right]=\left[\begin{array}{cc}
1 & T_{s} \\
0 & 1
\end{array}\right] \otimes\left[\begin{array}{c}
Z(k+1) \\
V_{z}(k)
\end{array}\right]+\left[\begin{array}{ccc}
0 & 0 & \frac{T_{s}^{2}}{2} \\
0 & 0 & T_{s}
\end{array}\right] \otimes\left[\begin{array}{l}
a_{x} \\
a_{y} \\
a_{z}
\end{array}\right]
$$

where $T_{s}$ represents the sampling interval, $a_{z}, a_{y}, a_{x}$ represents $\mathrm{x}, \mathrm{y}, \mathrm{z}$-axis acceleration respectively.

While time line moves to the moment where zero vertical velocity is detected by ZUPT, Kalman filter is triggered, updating vertical velocity to zero, and the previous non-zero vertical velocity feeds back to the Kalman filter as the offset to eliminate the accumulated error.

\subsection{Step Length Calculation}

The transition model $x(k)$ of the Kalman filter consists of vertical velocity, it is sin-like curve which crosses $\mathrm{x}$-axis twice in one step, and the zero points are the moments with maximum and minimum vertical acceleration which detected by ZUPT. The vertical velocity of trunk in one step (from one heel-touching-ground event (t1) to the next heel-touching-ground event (t2) is shown in Fig. 3.

The whole one step can be divided into two periods according to zero points ( $t 1$, $\mathrm{t} 2, \mathrm{t} 3$ ). The period from the start point ( $\mathrm{t} 1)$ to the middle zero point $(\mathrm{t} 2)$ represents the period from the first heel-touching-ground event to the stance event, that is, the period for the foot behind to catch the other foot which is in front. While the period from the middle zero point ( $\mathrm{t} 2$ ) to the end point $(\mathrm{t} 3)$ represents the period from the stance event to the second heel-touching-ground event, that is, the foot behind not only catches up the front foot (last period) but also opens up a new pace and it will become the front foot in next step.

At the beginning of walking, that is, the beginning of the first step, both feet are in stance state, waiting for opening up a new step. So there is no first period but the second period immediately, making vertical velocity to contain the period above the $\mathrm{x}$-axis only. The final step only contains first period but no second one. The foot 
Fig. 3 Vertical velocity of trunk in one step

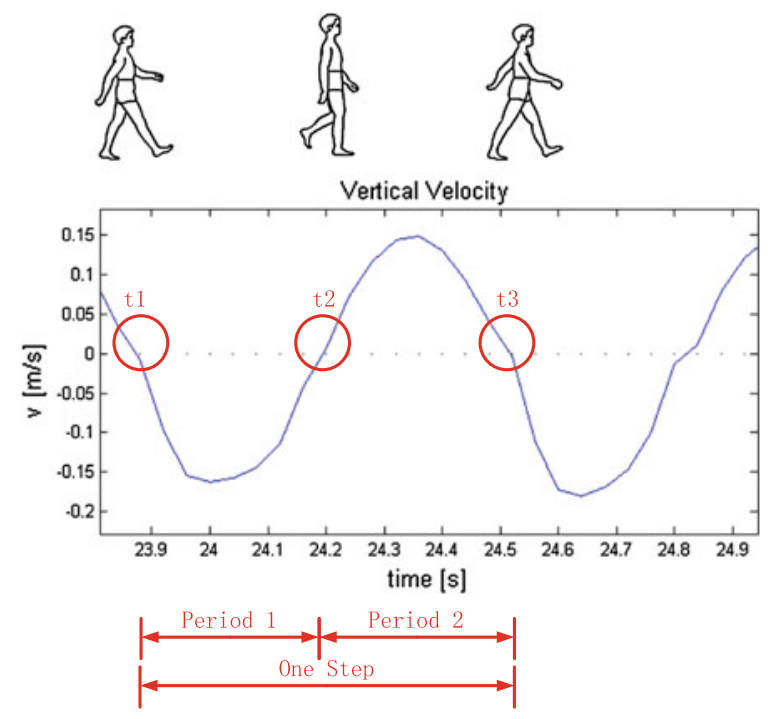

behind catches the front foot and stops once it has caught it up and the whole walking stops but not to open up new pace. So the graph of final step only contains the part below the x-axis.

So the vertical displacement of trunk in one step can be obtained through integrating the second period of the vertical velocity graph, and then taking the absolute value. Assuming the length of legs $L$ is known, we can obtain the step length $(D(k))$ through the Pythagorean Theorem by (4).

$$
D(k)=2 \sqrt{-2 L \int_{t 1}^{t 2} V_{z}(k)-\left(\int_{t 1}^{t 2} V_{z}(k)\right)^{2}}
$$

The method to obtain step length is also shown in Fig. 4. where $L$ represents the length of leg, $D$ is the step length, $h$ is the vertical displacement of trunk which can be obtained by (5).

$$
h(k)=-\int_{t 1}^{t 2} V_{z}(k)
$$

So the process we calculate step length is actually the process of calculating how far the foot behind is to catch up the other foot in front. The pace opened up by the first step is to be chased by the second step. So the first step should not be counted in (first step has no second period). The final step is not to open up a new pace but to chase the pace opened up by last step. So the final step should be counted in. 
Fig. 4 Vertical velocity of trunk in one step

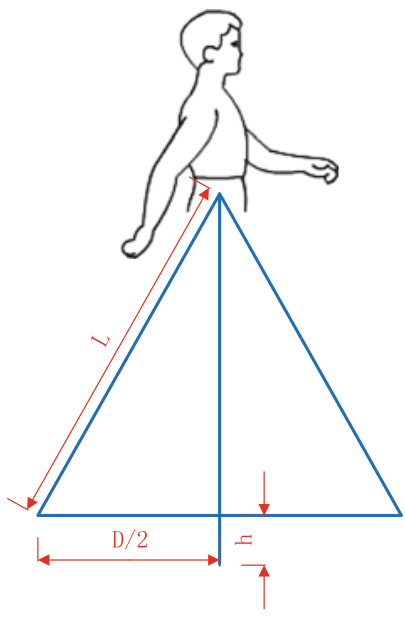

\subsection{Trajectory Calculation}

Gyroscope measured angular velocity is converted to Euler angles through the quaternion in real time to obtain the heading angle of each sampling point. Because we have obtained length of every step in previous job, what to do next is to calculate coordinate of every step. The method is shown in (6).

$$
\left[\begin{array}{l}
x(k+1) \\
y(k+1)
\end{array}\right]=\left[\begin{array}{l}
x(k) \\
y(k)
\end{array}\right]+D(k+1) \times\left[\begin{array}{c}
\sin (\operatorname{Yaw}(k+1)) \\
\cos (\operatorname{Yaw}(k+1))
\end{array}\right]
$$

where $x(k)$ and $y(k)$ represent the coordinate of steps, $D(k)$ represents the step length of the kth step, $\operatorname{Yaw}(k)$ represents the heading angle of kth step.

\section{Experiment and Results}

We use the Android smartphone as a carrier to acquire the phone built-in sensors data, to do real-time trajectory computing and to display the trajectory on screen. HTC Droid DNA is used in experiments, which is equipped with accelerometer BMA250, gyroscope InvenSense MPL Gyro, and virtual gravity sensor. The pedestrian in the experiment is a 24 years old male with leg length of $0.96 \mathrm{~m}$, and he walks through a building with the smartphone held on hand static to trunk. The data are recorded at $25 \mathrm{~Hz}$ clock rate. All the experiments were conducted at the 6th floor of No.2 Research Building in Haiyun Park of Xiamen University. The experimental data are record in text format and can be found at GitHub. ${ }^{1}$

\footnotetext{
${ }^{1}$ All the experimental data can be found at: https://github.com/ECG-XMU/SmartPhoneIndoorLocationDataset.
} 


\subsection{Simulation of ZUPT and Kalman Filter}

One experiment was conducted for detecting the two time of zero vertical velocity and to eliminate the drift error. The pedestrian in the experiment walked in a normal pace.

Using the data collected, the calculated vertical linear velocity and the moments ZUPT triggered were shown in Fig. 5.

We use +1 and -1 as the threshold of vertical linear acceleration to detect zero vertical velocity (the red line in the first graph of Fig. 5). As we can see in the second graph of Fig. 5, almost all the zero vertical velocity moments were detected (we use 1 to mark the heel-touch-ground event, and 2 to mark the stance event).

After obtaining zero vertical velocity moments, Kalman filter was applied to eliminate the drift error of sensors and to calculate the vertical velocity. We extract the calculated vertical velocity data of the experiment from beginning to about $22 \mathrm{~s}$ of time to show the power of ZUPT in Fig. 6.
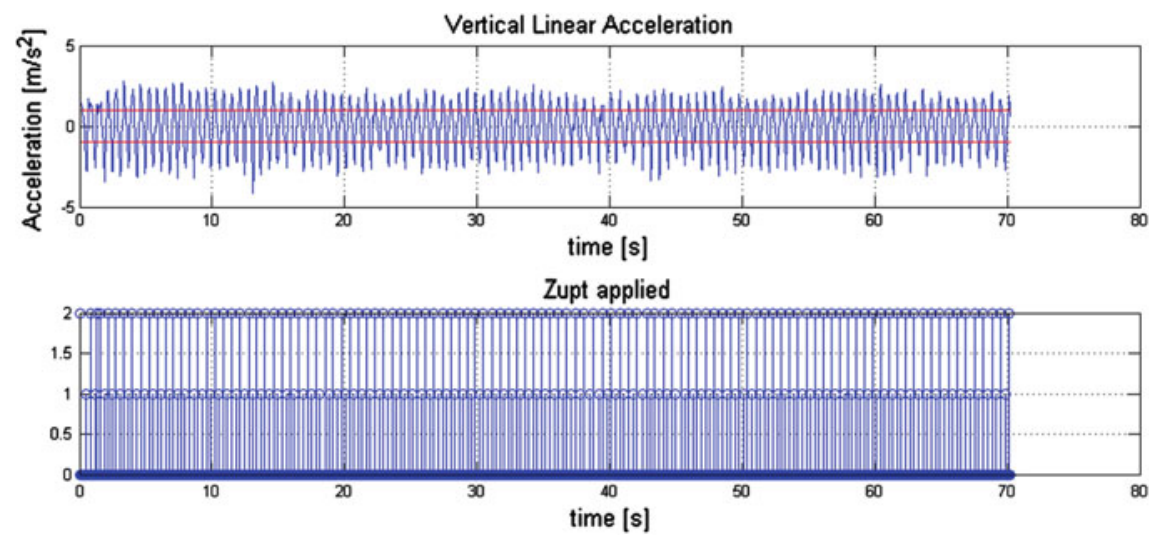

Fig. 5 The calculated vertical linear velocity and the moments ZUPT triggered

Fig. 6 Contrast between vertical velocity with ZUPT and without ZUPT

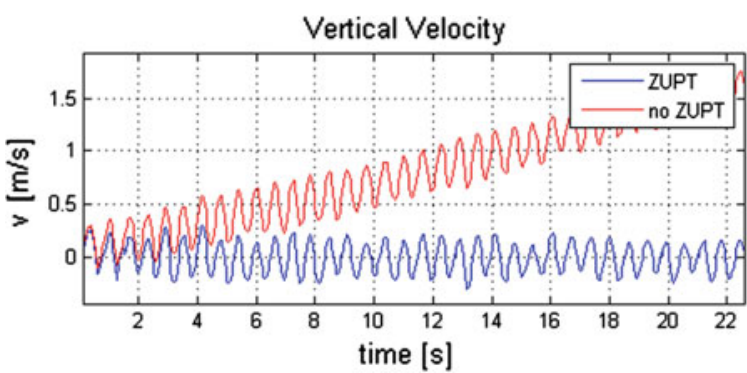


As shown in Fig. 6, the ZUPT limits the vertical velocity changing range around zero. It has successfully eliminated drift error of accelerator and greatly enhanced vertical velocity accuracy.

\subsection{Simulation of Step Length}

To verify the performance of the step length calculation, an experiment was carried out. The participant walked from the left side of the building to the right side of the building, then turned back and entered the room 605 . The whole path contains four turnings including two clockwise turnings and two anticlockwise turnings. Figure 7 shows calculated vertical velocity, the vertical displacement (integral of vertical velocity) and the step length of the experiment.

As can be seen from data in Fig. 7, vertical displacement of each step fell roughly between 0.025 and $0.05 \mathrm{~m}$, and length of each step fell about $0.55 \mathrm{~m}$, which is consistent with the physiological parameters with a male walking at normal speed whose leg length is $0.96 \mathrm{~m} \mathrm{[19].}$

Four phases marked in Fig. 7 represent four turnings along the path. The first two turnings are anticlockwise turnings and the other two are clockwise turnings. As shown in Fig. 7, the step lengths computed while making turnings were significantly smaller than the straight ones, this is consistent with the observation from daily walking.

After the first experiment, another set of three experiments were conducted under same environment to compare the distance of tracks measured by the system and the tape. The three experiments are walking in rectangle, straight and free walking. The distance data collected are presented in Table 1.

Fig. 7 Contrast between vertical velocity with ZUPT and without ZUPT
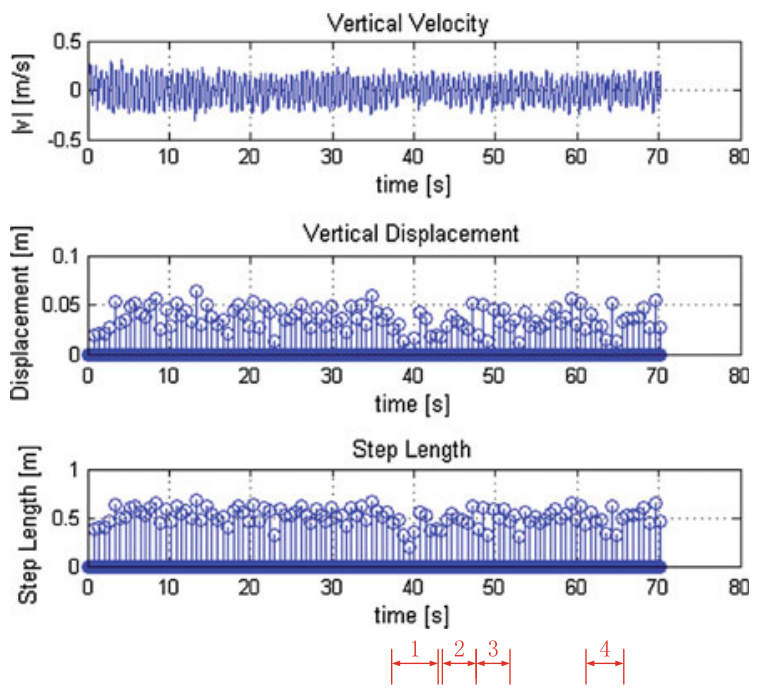
Table 1 The distance measured in given tracks

\begin{tabular}{l|l|l|l}
\hline & Rectangle & Straight & Free walking \\
\hline Real distance $(\mathrm{m})$ & 78 & 37.2 & 38.7 \\
\hline Distance calculated $(\mathrm{m})$ & 76.9485 & 36.7204 & 38.1859 \\
\hline Error rate $(\%)$ & 1.35 & 1.29 & 1.33 \\
\hline
\end{tabular}

where

$$
\text { Error rate }=\frac{\mid \text { True distance }- \text { Distance calculated } \mid}{\text { True distance }}
$$

According to the results, the maximum error rate is $1.35 \%$, and the average error rate is $1.32 \%$. So the distances tracked using the system matched the real track within a small variant.

\subsection{Experiment of Participant Navigation}

In this experiment, the participant held the smartphone on hand relatively stable to his trunk, walking from one side of 6th floor of No.2 Research Build straightly to the other side of building along the corridor. Then, the participant turned left (1), made a big turn till he changed the direction thoroughly and then kept walking. Before reaching the wall, he turned left (2) slightly until backed to the path he has walking. Then he turned right (3) to walk straightly on the previous path but only in the opposite direction. Finally, when he arrived at the door of room 605 , he turned

Fig. 8 Experiment of pedestrian navigation

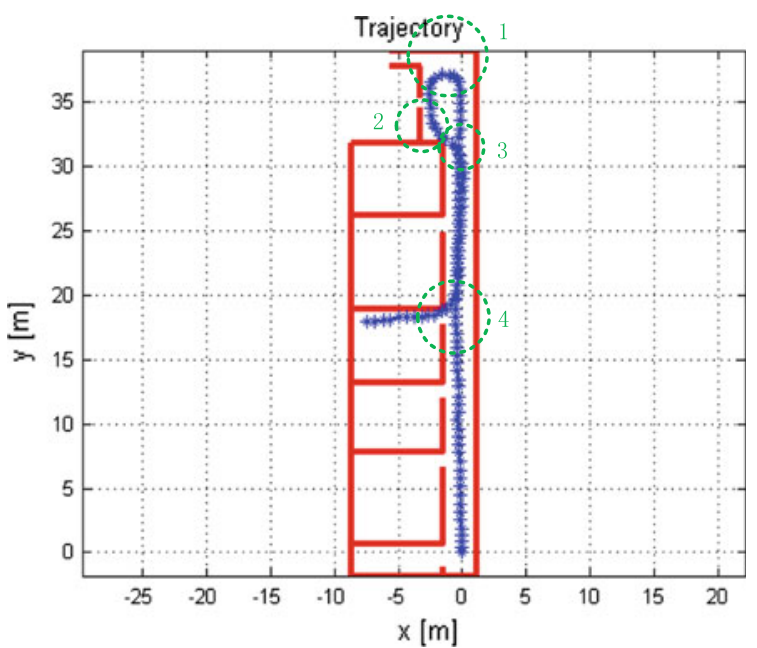


right (4) and entered the room, walked by the wall till he reached the far end of the room. The paths are shown in Fig. 8.

In the experiment, the distance of whole real track is $65.3 \mathrm{~m}$, and the distance of measured whole track is $64.3355 \mathrm{~m}$, the error is about $1.5 \%$. It can be concluded that the system achieved a reasonable accuracy and the measured track matches the real track.

\section{Conclusion}

This paper presented an algorithm for indoor positioning using a hand-held smartphone. We developed a method to detect the two times of zero vertical velocity through acceleration and gravity, and to correct the vertical velocity through a Kalman filter. The Pythagorean Theorem is applied to calculate the step length by vertical velocity. The main contribution of this paper is that we obtained high precision results with common hand-held smartphone common on market. The experimental results showed that we successfully achieved indoor positioning without any infrastructural information sources and this result demonstrated that the algorithm could be used in smartphone indoor navigation. Further work will be carried out to evaluation the system with more participants and in different indoor environments. The implementation of a downloadable mobile application will also be undertaken.

Acknowledgment This work was supported by the Natural Science Foundation of China (NSFC, No. 61201196).

\section{References}

1. Ojeda L, Borenstein J (2007) Personal dead-reckoning system for GPS-denied environments. In: Proceedings of IEEE international workshop safety, security rescue robot, pp 1-6

2. Alvarez JC, Gonzalez RC, Alvarez D, Lopez AM, Rodriguez-Uria J (2007) Multisensor approach to walking distance estimation with foot inertial sensing. In: Proceedings of IEEE 29th annual international conference engineering in medicine and biology society, pp 5719-5722

3. Dippold M (2006) Personal dead reckoning with accelerometers. In: Presented at the 3rd international forum applied wearable computing, Bremen, Germany

4. Eric F (2005) Pedestrian tracking with shoe-mounted inertial sensors. Proc IEEE Comput Graph Appl 25:38-46

5. Jimenez AR, Seco F, Prieto C, Guevara J (2009) A comparison of Pedestrian dead-reckoning algorithms using a low-cost MEMS IMU. In: Proceedings on IEEE international symposium on intelligent signal processing, pp 37-42

6. RaúlFeliz EZ, García-Bermejo JG (2009) Pedestrian tracking using inertial sensors. J Phys Agents 3:35-42 
7. Sagawa K, Inooka H, Satoh Y (2000) Non-restricted measurement of walking distance. In: Proceedings of IEEE international conference on systems, man, and cybernetics, vol 3, pp 1847-1852

8. Xiaoping Y, Bachmann ER, Moore H, Calusdian J (2007) Self-contained position tracking of human movement using small inertial/magnetic sensor modules. In: Proceedings of IEEE international conference on robotics and automation, pp 2526-2533

9. Alvarez D, Gonzalez RC, Lopez A, Alvarez JC (2006) Comparison of step length estimators from weareable accelerometer devices. In: Proceedings of IEEE 28th annual international conference of engineering in medicine and biology society, pp 5964-5967

10. Lei F, Antsaklis PJ, Montestruque LA, McMickell MB, Lemmon M, Yashan S, Hui F, Koutroulis I, Haenggi M, Min X, Xiaojuan X (2005) Design of a wireless assisted pedestrian dead reckoning system - the NavMote experience. IEEE Trans Instrum Meas 54(6):2342-2358

11. Shin SH, Park CG, Hong HS, Lee JM (2005) MEMS-based personal navigator equipped on the user's body. In: Presented at the ION GNSS 18th International Tech. Meeting Satellite Division, Long Beach, CA, USA, 13-16 Sep 2005

12. Weinberg H (2002) Using the ADXL202 in pedometer and personal navigation applications. Appl. Notes An-602, Analog Devices, Inc., Norwood, MA, USA

13. Renaudin V, Demeule V, Ortiz M (2013) Adaptative pedestrian displacement estimation with a smartphone. In: International conference on indoor positioning and indoor navigation, vol 12, pp 916-924

14. Kamisaka D, Muramatsu S, Iwamoto T et al (2011) Design and implementation of Pedestrian dead reckoning system on a mobile phone. IEICE Trans Inf Syst E94-D(6):1137-1145

15. Zhou B, Li Q, Mao Q, Tu W, Zhang X (2014) Activity sequence-based indoor pedestrian localization using smartphones. IEEE Trans Hum Mach Syst 1-13

16. Zheng L, Zhou W et al (2015) A foot-mounted sensor based 3D indoor positioning approach. In: IEEE twelfth international symposium on autonomous decentralized systems (ISADS), pp $145-150$

17. Zheng $\mathrm{X}$, Yang $\mathrm{H}$, Tang $\mathrm{W}$ et al (2014) Indoor Pedestrian navigation with shoe-mounted inertial sensors. Multimedia and Ubiquitous Engineering. Springer, Berlin, pp 67-73

18. Lan K-C, Shih W-Y (2014) Using smart-phones and floor plans for indoor location tracking. IEEE Trans Hum Mach Syst 44(2):211-221

19. Hunter J, Marshall R, McNair P (2004) Interaction of step length and step rate during sprint running. Med Sci Sports Exerc 36:261-271 